\title{
Evaluation of molnupiravir analogues as novel coronavirus (SARS-CoV-2) RNA-dependent RNA polymerase (RdRp) inhibitors - an in silico docking and ADMET simulation study
}

\author{
Necla KULABAŞ 1 (D), Tuğçe YEŞİL ${ }^{2}$ (D), İlkay KÜÇÜKGÜZEL ${ }^{1 *}$ (D) \\ 1 Department of Pharmaceutical Chemistry, Faculty of Pharmacy, Marmara University, Başıüyük 34854 İstanbul, \\ Turkey. \\ 2 Department of Pharmaceutical Toxicology, Faculty of Pharmacy, Marmara University, Başıüyük 34854 İstanbul, \\ Turkey. \\ * Corresponding Author. E-mail: ikucukguzel@marmara.edu.tr (İ.K.); Tel. +90-216 7775329
}

Received: 09 October 2021 / Accepted: 01 November 2021

\begin{abstract}
The severe acute respiratory syndrome coronavirus 2 (SARS-CoV-2), which is characterized by a wide range of symptoms including fever, dry cough, headache, decreased sense of taste and smell, was first identified in Wuhan, China in December 2019. Currently, the nucleoside analog, remdesivir has been approved for emergency use authorization (EUA) by the regulatory agencies for the treatment of COVID-19 patients. The need for new antiviral agents has been continuing due to the some disadvantages of remdesivir. Molnupiravir (MLN) that is developed for the treatment of hepatitis $\mathrm{C}$ virus (HCV), have been reported to show antiviral activity against SARS-CoV-2 according to the results of a high throughput screen of nucleoside analogs and also phase II/III clinical trials of MLN is ongoing. In this study, fifty four MLN analogs (twelve of them are found to be reported in the literature whereas forty two of them are novel molecules) against SARS-CoV-2 RdRp were designed and evaluated for their potential antiviral activity by using molecular modelling studies. While among the designed MLN analogs, compound C17 was found to have the best potential inhibitor with $-7.3 \mathrm{kcal} / \mathrm{mol}$ binding energy that is higher than molnupiravir and its active form EIDD-1931. Therefore, the isobutyric acid ester and monophosphate forms of C17 were also compared to the related MLN derivatives in terms of active site interactions. Lastly, the ten compounds with the best binding affinity including C17 were tested in silico for bioavailability, drug-likeness, ADME and safety profiles and were found to exhibit similar bioavailability and safety profile to MLN.
\end{abstract}

KEYWORDS: Molnupiravir; SARS-CoV-2 RdRp; ADMET prediction; docking studies.

\section{INTRODUCTION}

COVID-19 (human coronavirus) that is caused by coronavirus-2 (SARS-CoV-2), the cause of rapidly increasing numbers of severe pneumonia-like symptoms with fatal consequences, has spread rapidly around the world since December 2019. COVID-19 is the most disastrous disease in the recent years with more than one million deaths and millions infected, was declared a pandemic on $11^{\text {th }}$ March, 2020 by WHO and since then, 233,503,524 confirmed cases of COVID-19, including 4,777,503 deaths were reported [1]. All viruses, including SARS-CoV-2, change over time and any new mutations can potentially increase or decrease infectiousness and virulence. However several vaccines are available against SARS-CoV-2 strain, the numerous new SARS-CoV-2 variants have been detected and some of these variants including SARS-CoV-2 Alpha, Beta, Gamma, Delta, and Lambda may increase the risk of possible re-infection or reduced effectiveness of vaccination [2]. Besides all this, considering the negative economic and social effects of this pandemic which has arisen due to the mandatory isolation and quarantine of millions of people, the need for the development of specific antiviral agents is increasing day by day [3]. As computer-aided drug discovery provides significant advantage for the prediction of potential molecules even before synthesis, preclinical testing and clinical trials in several years, in silico drug design and development studies increases which focuses the treatment of COVID-19, the disaster needed urgent solution.

How to cite this article: Kulabaş N, Yeşil T, Küçükgüzel i. Evaluation of molnupiravir analogues as novel coronavirus (SARS-CoV-2) RNAdependent RNA polymerase (RdRp) inhibitors - an in silico docking and ADMET simulation study. J Res Pharm. 2021; 25(6): 967-981. 
To date, seven different human coronavirus strains which of two are alphacoronaviruses (229E and NL63 strains) and others are betacoronaviruses (OC43, HKU1, SARS, MERS, and SARS-CoV-2 strains) have been reported [4]. SARS-CoV-2 is a member of betacoronaviruses, such as the most aggressive strains SARS and MERS human coronaviruses with their remarkable mortality rates ( $10 \%$ and $36 \%$, respectively). SARS$\mathrm{CoV}-2$ genome encodes 16 non-structural proteins which are essential for the replication such as papain-like protease (nsp3), chymotrypsin-like main protease (3CL protease, nsp5), primase complex (nsp7-nsp8), RNAdependent RNA polymerase RdRp (nsp12), helicase (nsp13), and exoribonuclease (nsp14) [3]. Among these viral enzymes, RdRp is a crucial one, presenting an attractive target for the development of new antiviral agents against SARS-CoV-2 due to its essential role in viral RNA synthesis in the life cycle of RNA viruses. Furthermore, inhibition of the enzyme is not expected to cause target-related side effects due to the absence of a functional equivalent to $\mathrm{RdRp}$ in mammalian cells. The development of effective RdRp inhibitors to block viral replication has long been a research topic for many scientists. There are two known classes of RdRp inhibitors: nucleoside analogue inhibitors (NIs) and non nucleoside analogue inhibitors (NNIs) [5]. A nucleoside analogue known as remdesivir remains as the only drug approved by regulatory agencies to treat COVID-19. Due to the difficulty of synthesis, intravenous administration and cost, and failure in some clinical trials for their beneficial effects, efforts have focused on the development of new alternative inhibitors of SARS-CoV-2 replication. It has been reported that the mutagenic effects of known antiviral drugs and their analogues on SARS-CoV-2 were examined in many studies in the literature (Elfiky, 2020; Esam, 2022). Among them, molnupiravir (MLN, Synonyms: EIDD-2801; MK-4482), a prodrug, is a promising new drug targeting the coronavirus RNA polymerase based on results of reported many studies [6-9]. MLN is currently in phase II/III clinical trials based on encouraging preclinical data and its lack of toxicity and severe adverse side effects in phase I clinical trials [10].

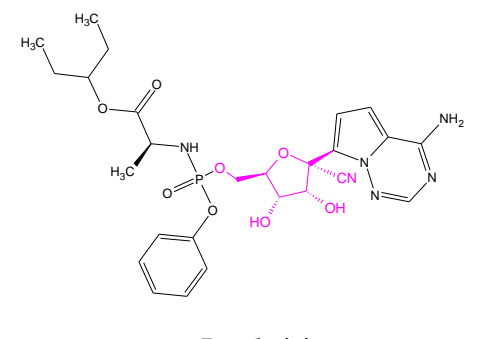

Remdesivir
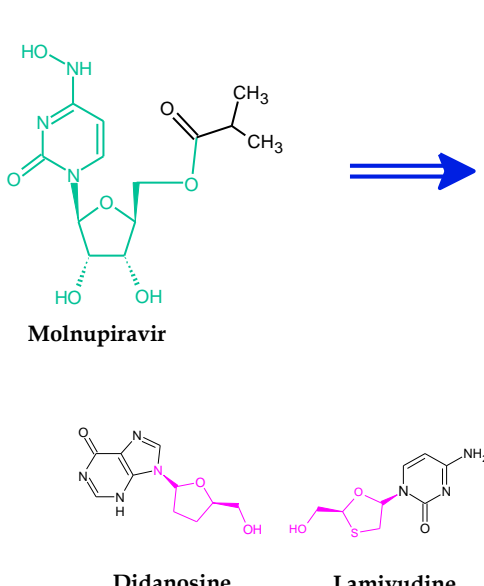

Didanosine
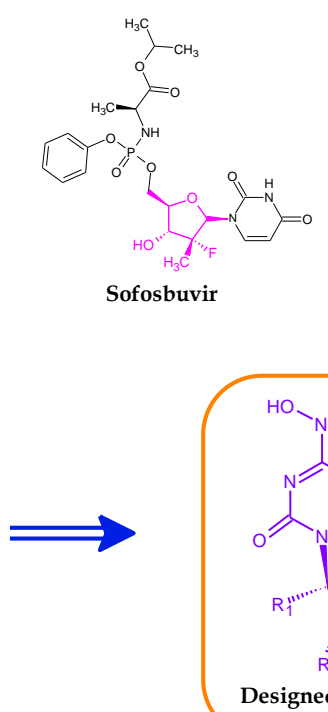

Lamivudine
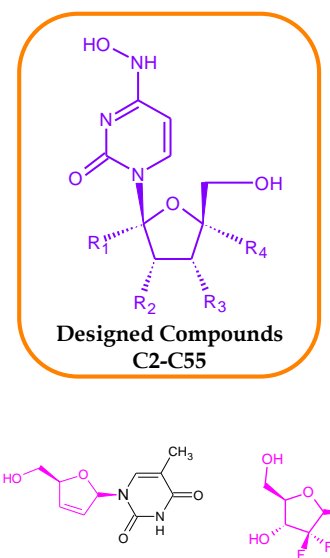

Stavudine

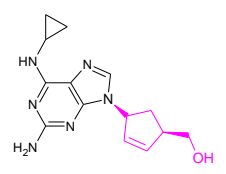

Abacavir

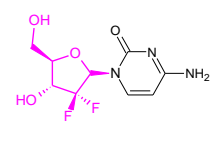

Gemcitabine
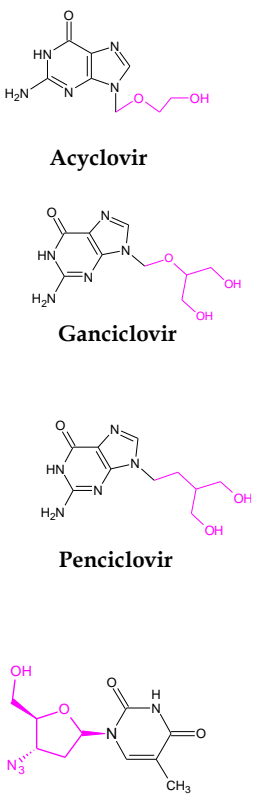

Zidovudine

Figure 1. Design of the novel molnupiravir analogs from the known antiviral drugs.

Molnupiravir is a prodrug of $\beta$-(D)-N $N^{4}$-hydroxycytidine (NHC) which is known as EIDD-1931 (also called as compound $\mathbf{C 1}$ in this article). The triphosphorylated derivative of EIDD-1931 is a substrate for viral RNA polymerases and interferes with viral replication. As known, cytidine is formed when cytosine is attached to a ribose ring and is a nucleoside molecule. The aim of this study was to design new analogs of molnupiravir analogs by making various deliberate modifications to the ribose ring of EIDD-1931 (Figure 1) and to evaluate the in silico inhibition potential of SARS-CoV-2 RdRp. The detailed ADME and safety profiles for the most active compounds identified in the screening were also calculated by in silico studies. 
For this purpose, we designed fifty four molnupiravir analogue compounds C2-C55 -twelve of them were reported as antiviral agents even compound C13 was patented to using in anti-COVID-19 therapy by Lili and Shaochun [11-17]- with various modifications on the EIDD-1931 by using the structures of known antiviral drugs. In our study, the in silico screening results of the designed compounds and their estimated drug potentials are discussed in detail.

\section{RESULTS and DISCUSSION}

\subsection{Molecular docking studies}

Nucleoside analogs which inhibit RdRp, i.e. molnupiravir and gemcitabine act as pyrimidine pseudobases after phosphorylation and cause irreversible mutations in the viral genome or inhibit the viral RdRp directly, in a concentration-dependent manner. The presence of a ribose ring in the chemical structure of nucleoside analogs are used as alternative substrates for endogenous nucleotides, causing mutations on the RNA chain, but it seems not to be crucial for antiviral activity [18]. Therefore, while designing our compounds, besides the modifications on the ribose ring, we also examined the active site interactions of the straight and branched chains instead of the ribose ring, as in the cases of acyclovir, ganciclovir and penciclovir. Moreover, we also evaluated the active site interactions of the 1,3-oxathiolane and cyclopentene rings instead of the ribose ring, as lamivudine and abacavir, respectively. In our study, we used SARS-CoV-2 RdRp enzyme structure $(6 \mathrm{~m} 71)$ the RNA strand free to evaluate the interactions of the designed MLN analogues (C2-C55) which has modifications on the ribose ring with the active site since known that nucleoside analogs RdRp inhibitors display mutagenic effect on the RNA strand after the phosphorylation.

The inhibition potentials of the designed and previously reported compounds (C2-C55) and molnupiravir against the SARS-CoV-2 RdRp enzyme were investigated using computer-aided methods. Compound C13 has been reported as anti-COVID-19 agent better than MLN by in vivo studies [16]. Dissimilar the previous study the active site interactions of C13 against SARS-CoV-2 RdRp were evaluated and discussed with the other designed compounds. When the results were evaluated, it was determined that all of the designed compounds showed quite significant affinity for binding to the active site of the enzyme and their binding energies were in the range of -7.3 to $-4.8 \mathrm{kcal} / \mathrm{mol}$ (see Supporting Information, Table S1). As a result of in silico screening, the binding energies of the 10 compounds with the highest potential enzyme inhibition and their interactions with the active site are given in Table 1. Moreover, these selected compounds C13-C15, C17-C19, C30, C32, C34 and C48 have higher binding energy than MLN.

Firstly we examined the interactions of the active form of molnupiravir, $\mathbf{C} 1$ with SARS-CoV-2 RdRp active site to compare with our designed compounds C2-C55. While $\beta$-D-N ${ }^{4}$-hydroxy group of cytidine showed two hydrogen bond with Thr556 residue as a donor, oxo group which is located at second position of cytidine ring, and nitrogen atom which is located at third position exhibited hydrogen bond interaction with Arg553 residue as an acceptor. Additionally, the hydroxyl group which is located at the fifth position of the ribose ring has two hydrogen bond interactions with Thr687 and Asn691 residues as donor and acceptor, respectively. Finally the distance between pyrimidine ring and Asp623 residue was found appropriate to pianion interaction (Figure 2D and 2E).

As a result of docking simulations, the best potential enzyme inhibitors were estimated as compounds C13-C15 and C17-C19 which are nucleoside analogues in which cyano group is introduced at 1' carbon of cytidine. Compound $\mathbf{C 1 7}$ has been found to have the highest affinity with a greater number of active site interactions and a binding energy of $-7.3 \mathrm{kcal} / \mathrm{mol}$. These results are better than the values calculated for previously reported anti-COVID-19 agents, C1 and C13 (Table 1). Predicted conformation of compound C17 superposed to $\mathbf{C} \mathbf{1}$ in complex with SARS-CoV-2 RdRp is given in Figure 2A. When we examined those interactions between $\mathrm{C17}$ and the active site of SARS-CoV-2 RdRp, we detected both two hydrogen bond interactions between 3'-hydroxy group of cytidine and Tyr619, Lys621 residues, and halogen bond interaction between 2'-fluoro atom of cytidine and Lys621 residue different from compound C1. Furthermore, the pyrimidine ring has exhibited pi-anion and pi-cation interaction with Arg553 and Asp623 residues, respectively (Figure 2B and 2C). Similarly for the other derivatives which possess cyano group, while substitution of the $3^{\text {th }}$ and/or $4^{\text {th }}$ positions of the ribose ring with fluorine and/or hydroxyl groups resulted in increased activity, the binding energy to the active site was found to be decreased for compound C16 which is a 3,4-dideoxy derivative (Table S1). 
Table 1. Interactions of the selected compounds with the active site of SARS-CoV-2 RdRp enzyme.

\begin{tabular}{|c|c|c|c|}
\hline Compounds & Binding Energy (kcal/mol) & $\begin{array}{c}\text { H-bond interactions } \\
\text { (Residue) }\end{array}$ & Distance $(\AA)$ \\
\hline \multirow{4}{*}{$\begin{array}{c}\text { C1 } \\
\text { (EIDD-2801) }\end{array}$} & \multirow{4}{*}{-6.0} & Arg553 & $2.24,2.40$ and 2.86 \\
\hline & & Thr556 & 2.75 and 3.13 \\
\hline & & Thr687 & 2.69 \\
\hline & & Asn691 & 2.29 \\
\hline \multirow{2}{*}{ C13 } & \multirow{2}{*}{-6.6} & $\operatorname{Arg} 555$ & 3.13 \\
\hline & & Asp623 & 4.37 \\
\hline \multirow{3}{*}{ C14 } & \multirow{3}{*}{-6.4} & Lys621 & 2.99 \\
\hline & & Cys 622 & 3.89 \\
\hline & & Asp623 & 3.81 \\
\hline \multirow{4}{*}{ C15 } & \multirow{4}{*}{-6.4} & Lys621 & 3.08 \\
\hline & & Cys 622 & 3.86 \\
\hline & & Asp623 & 3.85 \\
\hline & & Thr680 & 4.44 \\
\hline \multirow{7}{*}{ C17 } & \multirow{7}{*}{-7.3} & Asp452 & 2.30 \\
\hline & & Arg553 & 2.41 and 3.08 \\
\hline & & Ala554 & 2.22 \\
\hline & & Thr556 & 2.35 \\
\hline & & Tyr619 & 2.54 \\
\hline & & Lys621 & 2.75 and 2.55 \\
\hline & & Asp760 & 2.04 \\
\hline \multirow{4}{*}{ C18 } & \multirow{4}{*}{-6.5} & Asp452 & 4.20 \\
\hline & & Lys621 & 3.04 \\
\hline & & Cys 622 & 3.96 \\
\hline & & Asp623 & 3.84 \\
\hline \multirow{2}{*}{ C19 } & \multirow{2}{*}{-6.7} & Lys621 & 3.04 \\
\hline & & Asp623 & 3.84 \\
\hline \multirow{3}{*}{ C30 } & \multirow{3}{*}{-6.4} & Thr556 & 4.15 \\
\hline & & Thr680 & 4.44 \\
\hline & & Thr687 & 3.48 \\
\hline C32 & -6.5 & Thr556 & 3.46 and 3.51 \\
\hline \multirow{4}{*}{ C34 } & \multirow{4}{*}{-6.3} & Thr556 & 3.91 and 3.94 \\
\hline & & Cys 622 & 4.4 \\
\hline & & Thr680 & 4.33 \\
\hline & & Thr687 & 3.55 \\
\hline \multirow{2}{*}{ C48 } & \multirow{2}{*}{-6.5} & Lys621 & 3.04 \\
\hline & & Asp 623 & 3.92 \\
\hline \multirow{3}{*}{ C17_PD } & & Asp452 & 2.27 \\
\hline & -6.8 & Ala554 & 2.27 \\
\hline & & Thr556 & 2.39 \\
\hline & & Arg553 & 2.64 \\
\hline & & Thr556 & 2.62 \\
\hline MLN & -5.7 & Asn 691 & 2.50 \\
\hline & & Ser759 & 2.25 \\
\hline & & Asp760 & 2.45 \\
\hline & & Arg553 & 1.58 \\
\hline & & Ala554 & 2.31 \\
\hline C17_MP & -6.9 & Tyr619 & 2.80 \\
\hline & & Asp623 & 2.52 \\
\hline & & Arg553 & 2.32 and 2.85 \\
\hline & & Thr556 & 2.56 and 3.42 \\
\hline MLN_MP & -6.3 & Asn691 & 2.66 and 2.96 \\
\hline & & Ser759 & 2.58 \\
\hline
\end{tabular}

Abbreviations: MLN: Molnupiravir, C17-PD: prodrug form of compound C17, C17-MP: monophosphate derivatives of compound C17, MLN_MP: monophosphate derivatives of Molnupiravir. 


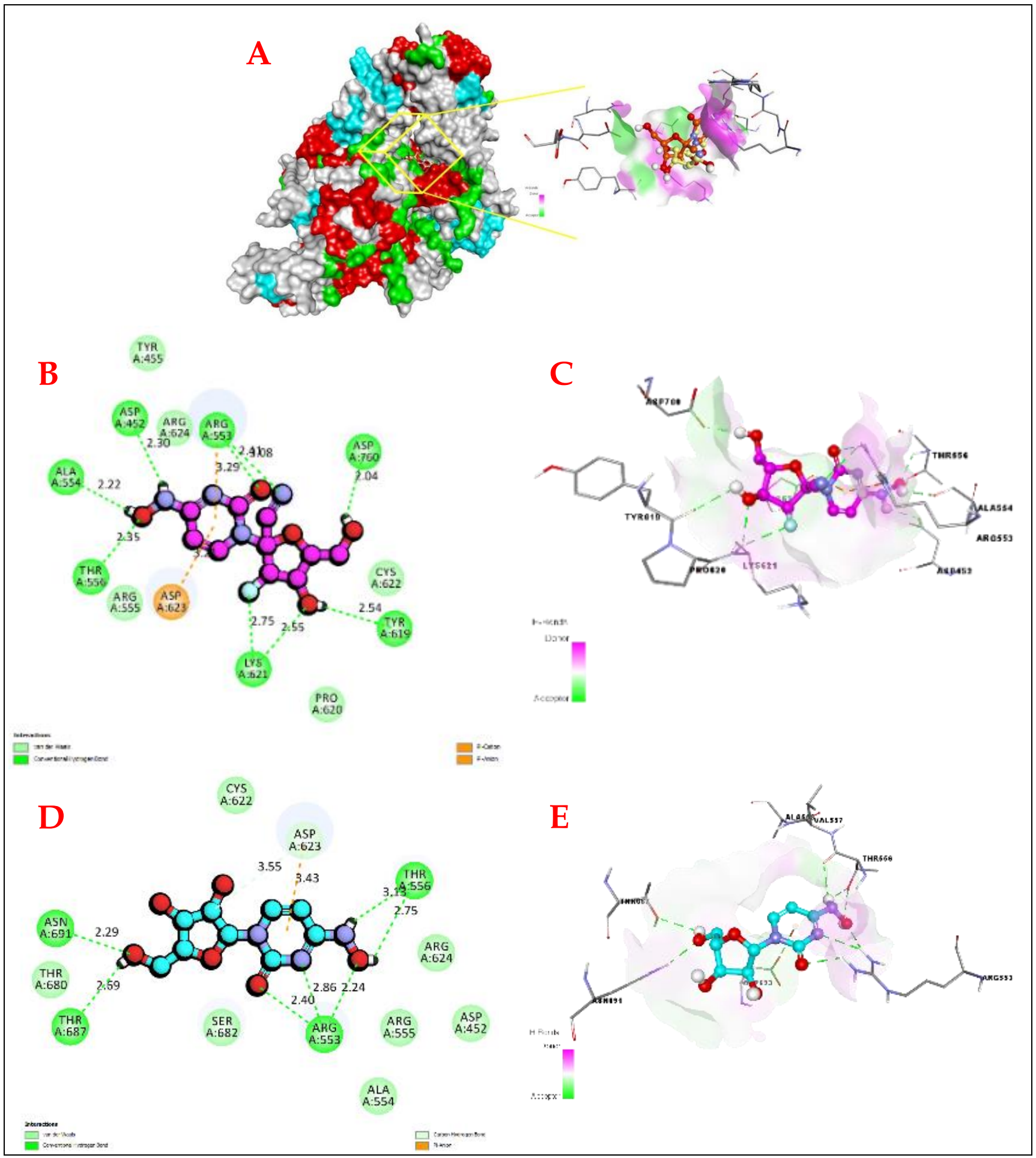

Figure 2. A) Predicted conformations of compound C17 (orange) superposed to C1 (yellow) in complex with SARS-CoV-2 RdRp. B) Interactions between compound C17 and SARS-CoV-2 RdRp as 2D diagram. C) Binding orientation of compound C17 in the active site of SARS-CoV-2 RdRp. D) Interactions between compound $\mathbf{C} 1$ and SARS-CoV-2 RdRp as 2D diagram. E) Binding orientation of compound $\mathbf{C 1}$ in the active site of SARS-CoV-2 RdRp.

As a result of docking simulations, the best potential enzyme inhibitors were estimated as compounds C13-C15 and C17-C19 which are nucleoside analogues in which cyano group is introduced at 1 ' carbon of cytidine. Compound $\mathbf{C 1 7}$ has been found to have the highest affinity with a greater number of active site interactions and a binding energy of $-7.3 \mathrm{kcal} / \mathrm{mol}$. These results are better than the values calculated for previously reported anti-COVID-19 agents, C1 and C13 (Table 1). Predicted conformation of compound C17 
superposed to $\mathbf{C} \mathbf{1}$ in complex with SARS-CoV-2 RdRp is given in Figure 2A. When we examined those interactions between $\mathrm{C17}$ and the active site of SARS-CoV-2 RdRp, we detected both two hydrogen bond interactions between 3'-hydroxy group of cytidine and Tyr619, Lys621 residues, and halogen bond interaction between 2'-fluoro atom of cytidine and Lys621 residue different from compound C1. Furthermore, the pyrimidine ring has exhibited pi-anion and pi-cation interaction with Arg553 and Asp623 residues, respectively (Figure 2B and 2C). Similarly for the other derivatives which possess cyano group, while substitution of the $3^{\text {th }}$ and/or $4^{\text {th }}$ positions of the ribose ring with fluorine and/or hydroxyl groups resulted in increased activity, the binding energy to the active site was found to be decreased for compound C16 which is a 3,4-dideoxy derivative (Table S1).

Among the most active compounds, C30, C32 and C34 contain an azido group on the 4' carbon of cytidine. While these compounds exhibited similar hydrogen bonding interactions to other designed cytidine derivatives, the azido group has been located in the distance that can make the charge transfer interaction with Asp760 residue which identifies the active site. This contribution increases the affinity into the active site. The in silico binding affinities of compounds C39, C45 and C48 decrease when the ribose ring is replaced by the 1,3-oxathiolane ring. Nevertheless, compound C48 has exhibited increased inhibitor effect due to hydrogen bond interaction between cyano group and Asp623 residue, when the cyano group is at the fifth position of 1,3-oxathiolane ring.

While compounds C20-C28 which carrying chloro methyl group on the 4' carbon atom of cytidine shown that similar active site interactions to compounds C2-C12, when the ribose ring is replaced by the cyclopentene ring, substitution with azido and cyano group caused increased binding affinity. In addition to, although the other our designed compounds which have straight and branched chains instead of ribose, exhibit significant interactions with the active site of the target enzyme, the presence of a 5-membered cyclic ring was found to be essential for activitydue to decreased binding affinity.

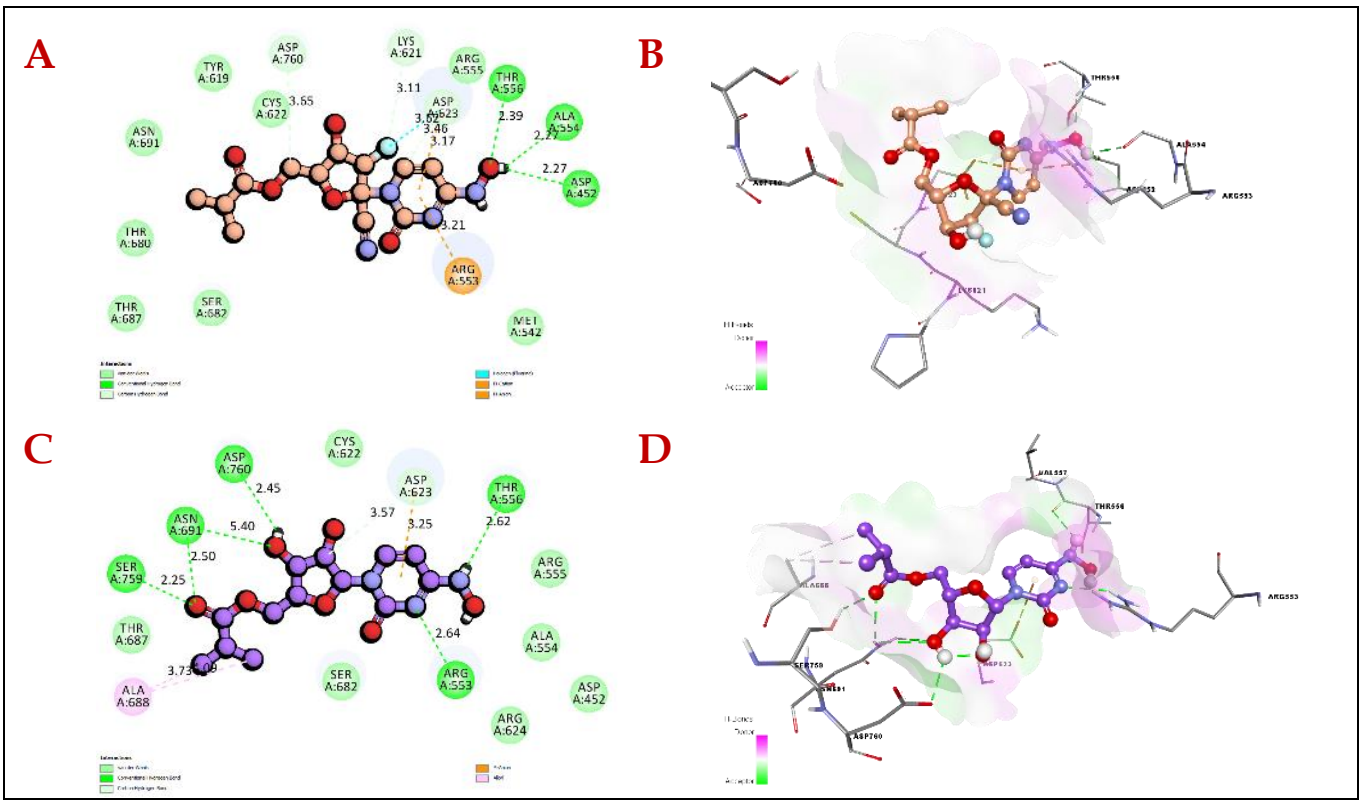

Figure 3. A) Interactions between compound C17_PD and SARS-CoV-2 RdRp as 2D diagram. B) Binding orientation of compound C17_PD in the active site of SARS-CoV-2 RdRp. c) Interactions between compound MLN and SARS-CoV-2 RdRp as 2D diagram. D) Binding orientation of compound MLN in the active site of SARS-CoV-2 RdRp.

As known, MLN is a prodrug and isobutyric acid ester form of $\mathbf{C 1}$. After determination of the most active compound $\mathbf{C 1 7}$ according to the molecular docking results, isobutyric acid ester form of C17 (C17_PD) was designed to evaluate the SARS-CoV-2 RdRp inhibition potential of the possible unchanged prodrug molecule. The interactions of both MLN and C17_PD with the active site are given Figure 3. In spite of hydrogen bonding interactions were detected between ester groups $(\mathrm{C}=\mathrm{O})$ of $\mathbf{M L N}$ and Asn691 and Ser759 residues, the mentioned interactions disappeared for C17_PD in comparison with active form C17. These findings are also supported by decreased binding energy. 
Finally, as MLN undergoes triphosphorylation after hydrolysis of the ester group to show its mutagenic effect on the RNA strain, the monophosphate derivatives of both MLN (MLN_MP) and C17 (C17_MP) were docked into the target active site and compared for their binding affinity (Figure 4). While the hydrogen bonding interactions of MLN_MP with Asn691 and Ser759 residues are continuing, monophosphate moiety of C17_MP interacted with Asp623 as a hydrogen bond acceptor besides Van der Waals interactions with Asp760 residue.

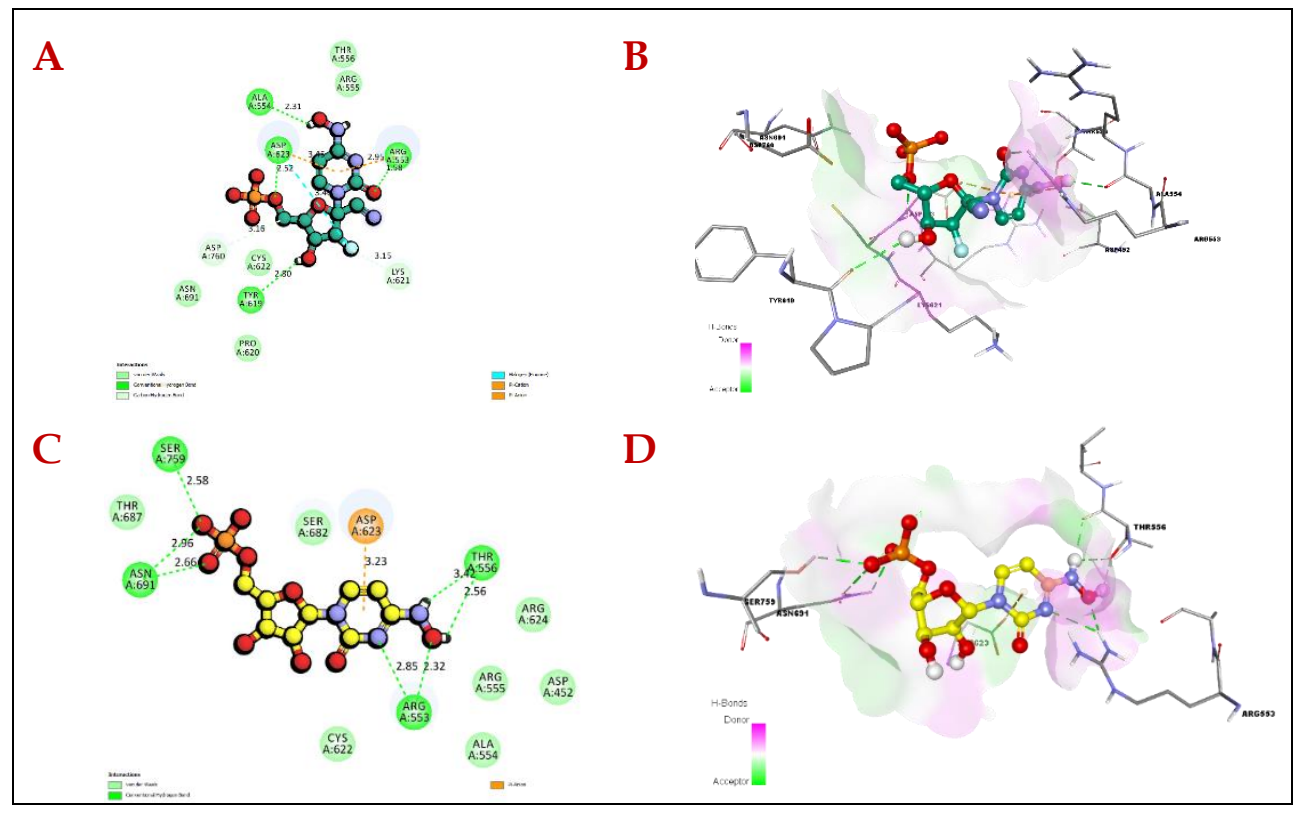

Figure 4. A) Interactions between compound C17_MP and SARS-CoV-2 RdRp as 2D diagram. B) Binding orientation of compound C17_MP in the active site of SARS-CoV-2 RdRp. c) Interactions between compound MLN_MP and SARS-CoV-2 RdRp as 2D diagram. D) Binding orientation of compound MLN_MP in the active site of SARS-CoV-2 RdRp.

The obtained docking results have shown that, compound C17 which has been designed in this study, is the best candidate, and its prodrug C17_PD and the monophosphate derivative C17_MP exhibits more inhibitory potential than both MLN and compound C1_MP.

\subsection{Analysis of bioavailability, drug-likeness and ADME/T profile}

Computational methods present an advantage of being able to estimate bioavailability and safety of drug candidates and to select better lead compounds even before they are synthesized and undergo preclinical tests and clinical trials [19].

\subsubsection{Analysis of bioavailability and drug-likeness}

As well known, it is the main issue for effectiveness that a drug molecule must reach the target in sufficient concentration. Molecular weight (MW), number of hydrogen acceptors (nON), number of hydrogen donors (nOHN), number of rotatable bonds ( nRot), topological polar surface area (TPSA), octanol-water partition coefficient ( $\left.\mathrm{Log} \mathrm{P}_{\mathrm{ow}}\right)$ and aqueous solubility $(\mathrm{LogS})$ are main characteristics that affect membrane permeability, lipophilicity and solubility therefore bioavailability and drug-likeness of a compound. The physico-chemical, pharmacokinetic, drug-likeness and related parameters of the compounds in this study were evaluated by using the SwissADME Web tool. Table 2 lists bioavailability and druglikeness characteristics of our compounds selected as they showed more interactions with the active site of the SARS-CoV-2 RdRp enzyme (please find Table S2 supports bioavailability and drug-likeness characteristics of all compounds in this study). MW of all compounds were found lower than $500 \mathrm{Da}$ and the MW of C17 (which is the most potent compound in the study) is $286.22 \mathrm{~g} / \mathrm{mol}$ and also, MW of C17_PD (prodrug of C17) is $356.31 \mathrm{~g} / \mathrm{mol}$ (MW of MLN:329.31 g/mol and MW of EIDD-1931, active form of MLN:259.22 g/mol). Other physicochemical properties (nON, nOHN, nRot, TPSA) of the C17_PD were found to show similarity with MLN as such in similarity of C17 with EIDD-1931. All selected compounds 
were estimated to have high aqueous solubility. None of the selected compounds exceed the range limit of LogP. Also, C17 and C17_PD were found to have better lipophilicity (LogP) than EIDD-1931 and MLN.

There are different type rule-based approaches often originating from analyses by major pharmaceutical companies aiming to improve the quality of their proprietary chemical collections; and two of them the Lipinski (Pfizer) and the Muegge (Bayer) methods were used to evaluate the drug-likeness of the compounds in this study. Therefore, selection criteria for an ideal drug candidate molecule according to rules established by Lipinski et al. [20] are that $\mathrm{MW}<500 \mathrm{Da}, \log \mathrm{P}<5, \mathrm{nON} \leq 10, \mathrm{nOHN} \leq 5$ and according to rules established by Muegge et al. [21] are that $200 \leq \mathrm{MW} \leq 600,-2 \leq \log \mathrm{P} \leq 5$, TPSA $\leq 150 \AA^{2}$, number of rings $\leq 7$, number of $\mathrm{C}>4$, number of heteroatom $>1, \mathrm{nROT} \leq 15, \mathrm{nON} \leq 10, \mathrm{nOHN} \leq 5$. Compounds violating more than one of these rules can pose low oral bioavailability. Only, C13 was found to exhibit 2 violations and other compounds have been found with one or less violation. C17_PD and C17 were found to have no violation.

Table 2. List of molecular properties (physico-chemical, lipophilicity, water solubility, drug likeness) of the selected compounds by using online webserver SwissADME.

\begin{tabular}{|c|c|c|c|c|c|c|c|c|c|}
\hline \multirow[b]{2}{*}{ Compound } & \multicolumn{5}{|c|}{ Physicochemical Properties } & \multirow{2}{*}{$\begin{array}{c}\text { Lipophilicity } \\
\operatorname{LogP}_{\mathrm{o} / \mathrm{w}}\end{array}$} & \multirow{2}{*}{$\begin{array}{c}\begin{array}{c}\text { Water } \\
\text { solubility }\end{array} \\
\text { LogS } \\
(E S O L)\end{array}$} & \multicolumn{2}{|c|}{ Drug-Likeness } \\
\hline & $\begin{array}{c}\text { MW } \\
\text { (g/mol) }\end{array}$ & $\mathrm{nON}$ & nOHN & nRot & $\begin{array}{c}\text { TPSA } \\
\left(\AA^{2}\right)\end{array}$ & & & $\begin{array}{c}\text { Lipinski } \\
\text { Rule } \\
\text { nviol }\end{array}$ & $\begin{array}{c}\text { Muegge } \\
\text { Rule } \\
\text { nviol }\end{array}$ \\
\hline $\begin{array}{c}\text { C1 } \\
\text { (EIDD-2801) }\end{array}$ & 259.22 & 7 & 5 & 3 & 137.07 & -1.82 & -0.48 & 0 & 0 \\
\hline $\mathrm{C} 13$ & 284.23 & 8 & 5 & 3 & 160.86 & -2.27 & 0.01 & 0 & 2 \\
\hline C14 & 268.23 & 7 & 4 & 3 & 140.63 & -1.54 & -0.18 & 0 & 1 \\
\hline C15 & 268.23 & 7 & 4 & 3 & 140.63 & -1.56 & -0.18 & 0 & 1 \\
\hline $\mathrm{C} 17$ & 286.22 & 8 & 4 & 3 & 140.63 & -1.31 & -0.31 & 0 & 1 \\
\hline C18 & 286.22 & 8 & 4 & 3 & 140.63 & -1.34 & -0.31 & 0 & 1 \\
\hline C19 & 304.21 & 9 & 4 & 3 & 140.63 & -1.02 & -0.57 & 0 & 0 \\
\hline C30 & 314.25 & 10 & 5 & 4 & 186.82 & -1.68 & -0.63 & 1 & 1 \\
\hline C32 & 302.22 & 10 & 4 & 4 & 166.59 & -1.11 & -1.10 & 1 & 1 \\
\hline C34 & 316.25 & 10 & 4 & 4 & 166.59 & -0.70 & -1.29 & 1 & 1 \\
\hline $\mathrm{C} 48$ & 270.27 & 6 & 3 & 3 & 145.70 & -0.78 & -0.49 & 0 & 0 \\
\hline MLN & 329.31 & 8 & 4 & 6 & 143.14 & -0.88 & -0.83 & 0 & 0 \\
\hline C17-PD & 356.31 & 9 & 3 & 6 & 146.70 & -0.31 & -1.17 & 0 & 0 \\
\hline
\end{tabular}

Abbreviations: MW: Molecular weight; nON: Number of hydrogen acceptors; $n O H N$ : Number of hydrogen donors; $n$ Rot: Number of rotatable bonds; TPSA: Topological polar surface area, LogPo/w: Octanol-water partition coefficient; LogS (ESOL): Estimating aqueous solubility from molecular structure; MLN: Molnupiravir, C17_PD: prodrug form of compound C17.

\subsubsection{Analysis of ADME and toxicity profile parameters}

The computational models that can predict ADMET profiles have became an alternative and complementary approach to existing in vitro and in vivo toxicity tests, thereby minimizing the need for animal testing, reducing the cost and time of relevant tests, and improving bioavailability/toxicity prediction and efficacy/safety assessments [19,22]. The ADMET profile of a xenobiotic is in a close relationship with bioavailability and drug-like properties, however findings were presented in the different sections and tables to discuss easily. pkCSM [23], eMolTox, ProToxII and admetSAR, fee free open sources and comprehensive computer readable databases, are tools that we used to predict ADME/T-associated properties of our compounds selected due to their superior interactions with the active site of the SARSCoV-2 RdRp enzyme. Also, SwissADME [24] and Molinspiration web tools and OSIRIS data warrior software [25] were applied to evaluate absorption and medicinal chemistry profiles (volume of molecules, drug-likeness score etc.) of the selected compounds. 
Table 3. Absorption, Distribution, Metabolism and Excretion (ADME) and medicinal chemistry properties of the selected compounds.

\begin{tabular}{|c|c|c|c|c|c|c|c|c|c|c|c|c|c|c|}
\hline & Parameters & $\mathrm{C} 13$ & C14 & C15 & $\mathrm{C} 17$ & $\begin{array}{l}\text { C17- } \\
\text { PD }\end{array}$ & C18 & C19 & C30 & C32 & C34 & $\mathrm{C} 48$ & MLN & $\begin{array}{c}\text { EIDD } \\
-1931 \\
\text { (C1) }\end{array}$ \\
\hline \multirow{6}{*}{ 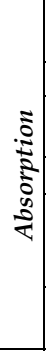 } & $\begin{array}{l}\text { Caco2 } \\
\text { permeability }\end{array}$ & 0.377 & 0.077 & 0.424 & 0.073 & 0.428 & 0.442 & 0.446 & 0.347 & 0.05 & 0.045 & -0.007 & 0.498 & 0.425 \\
\hline & GI absorbtion $^{a}$ & low & low & low & low & low & low & low & low & low & low & low & low & low \\
\hline & $\begin{array}{l}\text { P-glycoprotein } \\
\text { substrate }^{b}\end{array}$ & no & no & no & no & no & no & no & no & no & no & no & no & no \\
\hline & Volumec & 227.5 & 219.46 & 219.46 & 224.42 & 294.32 & 224.42 & 229.02 & 251.77 & 232.45 & 248.69 & 212.74 & 280.87 & 210.97 \\
\hline & \begin{tabular}{l|} 
Human \\
intestinal \\
absorbtion $^{a}(\%)$ \\
\end{tabular} & 46 & 58.334 & 61.277 & 55.033 & 57.528 & 55.006 & 61.484 & 47.488 & 55.331 & 56.163 & 62.618 & 52.006 & 39.275 \\
\hline & $\begin{array}{l}\text { Bioavailibility } \\
\text { score }\end{array}$ & 0.55 & 0.55 & 0.55 & 0.55 & 0.55 & 0,55 & 0.55 & 0.11 & 0.11 & 0.11 & 0.55 & 0.55 & 0.55 \\
\hline \multirow{3}{*}{ 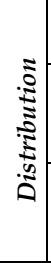 } & $\begin{array}{l}\text { VDss (human, } \\
(\log \mathrm{L} / \mathrm{kg})\end{array}$ & -0.525 & -0.789 & -0.561 & -0.749 & 0.282 & -0.472 & -0.709 & -0.27 & -0.495 & -0.476 & -0.682 & 0.847 & -0.147 \\
\hline & $\begin{array}{l}\text { BBB } \\
\text { permeability } \\
(\log \text { BB })\end{array}$ & -1.345 & -1.248 & -1.129 & -1.396 & -1.155 & -1.245 & -1.292 & -1.565 & -1.648 & -1.67 & -1.136 & -1.02 & -1.225 \\
\hline & $\begin{array}{l}\text { CNS } \\
\text { permeability }{ }^{a} \\
(\log \text { PS) }\end{array}$ & -4.186 & -3.745 & -3.385 & -3.742 & -3.335 & -3.381 & -3.42 & -3.516 & -3.451 & -3.432 & -3.304 & -3.739 & -4.124 \\
\hline \multirow{6}{*}{ 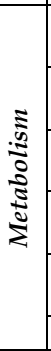 } & $\begin{array}{l}\text { CYP1A2 } \\
\text { substrate }\end{array}$ & no & no & no & no & no & no & no & no & no & no & no & no & no \\
\hline & $\begin{array}{l}\text { CYP2C19 } \\
\text { substrate }\end{array}$ & no & no & no & no & no & no & no & no & no & no & no & no & no \\
\hline & $\begin{array}{l}\text { CYP2C9 } \\
\text { substrate }\end{array}$ & no & no & no & no & no & no & no & no & no & no & no & no & no \\
\hline & $\begin{array}{l}\text { CYP2D6 } \\
\text { substrate }\end{array}$ & no & no & no & no & no & no & no & no & no & no & no & no & no \\
\hline & $\begin{array}{l}\text { CYP3A4 } \\
\text { substrate }\end{array}$ & yes & yes & yes & yes & no & yes & yes & yes & yes & yes & yes & yes & no \\
\hline & UGT catayzed & no & no & no & no & yes & yes & yes & no & no & yes & no & yes & no \\
\hline \multirow{2}{*}{ 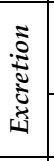 } & $\begin{array}{l}\text { Total Clearance } \\
(\log \\
\mathrm{ml} / \mathrm{min} / \mathrm{kg})\end{array}$ & 0.603 & 0.607 & 0.612 & 0.524 & 0.088 & 0.512 & 0.601 & -0.185 & -0.161 & -0.21 & 0.211 & 0.175 & 0.525 \\
\hline & \begin{tabular}{l|}
$\begin{array}{l}\text { Renal OCT2 } \\
\text { substrate }\end{array}$ \\
\end{tabular} & no & no & no & no & no & no & no & no & no & no & no & no & no \\
\hline \multirow{3}{*}{ 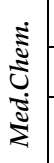 } & $\mathrm{PAINS}^{b}$ & 0 & 0 & 0 & 0 & 0 & 0 & 0 & $1^{*}$ & $1^{*}$ & $1^{*}$ & 0 & 0 & 0 \\
\hline & Brenk $^{b}$ & $1^{* *}$ & $1^{* *}$ & $1^{*^{*}}$ & $1^{* *}$ & $1^{* *}$ & $1^{* *}$ & $1^{* *}$ & $4^{* * *}$ & $4^{* * *}$ & $4^{* * *}$ & $1^{* *}$ & $1^{* *}$ & $1^{* *}$ \\
\hline & Drug-likeness ${ }^{d}$ & -7.48 & -5.69 & -8.79 & -6.83 & -5.8536 & -6.65 & -10.07 & 1.32 & -1.97 & -1.18 & -4.73 & -2.53 & -1.89 \\
\hline
\end{tabular}

${ }^{a}$ These studies were performed by using online webserver pkCSM; ${ }^{b}$ These studies were performed by using online webserver SwissADME; ${ }^{c}$ This parameter calculated by using Molinspiration; " These studies were performed by using OSIRIS data warrior software. PAINS ALERTS: *azo group BRENK ALERTS: ** oxygen-nitrogen-single bond; ${ }^{* * *}$ azido group, diazo group, oxygen-nitrogen-single bond, quaternary nitrogen

\section{ADME}

P-glycoprotein (P-gp) that is a member of ABC transporter family, actively pumps xenobiotics out of the cells and plays an important role in the intestinal absorption and excretion of drugs and also, limits various xenobiotics enter into the central nervous system (CNS). Therefore, efficacy and safety of drug molecules related to being P-gp substrates, inhibitors or inductors [26]. The selected compounds were not predicted to be a P-gp substrate (Table 3) or inhibitor (Table 4) like MLN and active form of MLN (EIDD1931). The human colon epithelial cancer cell line, Caco-2, is commonly used as a prediction model of human intestinal absorption and permeability of xenobiotics [27]. The selected compounds exhibited low Caco-2 permeability and human intestinal absorption (Table 3). Bioavailability scores of the selected compounds except C30, C32 and C34 were found to be similar to MLN and EIDD-1931 (Table 3).

The volume of distribution (VDss) is the theoretical value to present the distribution profile of a molecule and higher values means drug molecules distributed to tissues rather than plasma. VDss of all the selected compounds were found low and the predicted distribution profile of C17 and C17_PD shows similarity to EIDD-1931 and MLN, respectively (Table 3). The blood-brain barrier (BBB) and central nervous system (CNS) permeability of the drugs except CNS targeted drugs due to cause neurotoxicity is not expected [28]. The selected compounds have been estimated to be poorly BBB permeable and unable to penetrate the CNS (Table 3). 
All selected compounds except C17_PD (due to isobutyric acid ester form of C17) were predicted to be substrates of CYP3A4 metabolism enzyme that is responsible for metabolism of many drugs (Table 3).

Total clearance refers to the combination of hepatic and renal clearance of a molecule and predicted total clearance values of the selected compounds were shown in Table 3 in $\log \mathrm{ml} / \mathrm{min} / \mathrm{kg}$. Also, compounds were tested to be a renal OCT2 substrate and none of the compounds have been expected to be a renal OCT2 substrate according to the pkCSM prediction tool.

\section{Toxicity}

The compounds selected according to their highest affinity prediction with the active site of the SARSCoV-2 RdRp, were evaluated in silico for different hazard endpoints, structural alerts, disruption potential on Tox21 pathways, target organ toxicity potential and inhibition potentials to major drug metabolism enzymes and drug transporters. Table 4 lists the predicted toxicity properties of the selected compounds.

$\mathrm{LD}_{50}$ refers to the degree of acute toxicity of xenobiotics and corresponds to doses that are likely to kill $50 \%$ of the animals in a batch used for experimentation. Therefore, $\mathrm{LD}_{50}$ value is an important parameter to classify the toxicity of a compound. Both oral rat acute toxicity $\left(\mathrm{LD}_{50}, \mathrm{~mol} / \mathrm{kg}\right)$ values (predicted by online web server pkCSM) and predicted $\mathrm{LD}_{50}(\mathrm{mg} / \mathrm{kg}$ ) values (predicted by online web server Protox-II) of the selected compounds were shown in Table 4 . All compounds were classified in category 4 according to the GHS classification (Category IV; chemicals with $300<\mathrm{LD}_{50} \leq 2000 \mathrm{mg} / \mathrm{kg}$ ) [29]. LOAEL refers to the low observed adverse effect level of the xenobiotics in the preclinic chronic toxicity studies and predicted LOAEL values of the selected compounds were shown in Table 4. Predicted LOAEL values of the C17 and C17_PD are $2.083 \log \mathrm{mg} / \mathrm{kg} \_b w /$ day and $2.824 \mathrm{og} \mathrm{mg} / \mathrm{kg} \_b w /$ day, respectively.

None of the compounds is expected to exhibit cytotoxicity according to the in silico test results (Table 4). Predicted AMES mutagenicity test results of the selected compounds were positive, unlike MLN and EIDD-1931. All selected compounds, MLN and EIDD-1931 were predicted to have DNA damage potential (genotoxicity) and also C17_PD were found inactive for the carcinogenicity potential similar to MLN (Table 4). Structural alerts (also known as toxicophores/toxic fragments) are chemical substructures that indicate or associate to specific toxic endpoints and are widely accepted in chemical toxicology and regulatory decisions. Brenk and Pan Assay Interference Compounds (PAINS) filters in the SwissADME web tool were applied and also structural alerts in the MolTox web tool were scanned. Compounds C30, C32 and C34 were predicted to contain four Brenk alerts and one PAINS alert related to the reactivity potential of the azido group on the 4' carbon of cytidine. On the other hand, C17 and C17_PD exhibited no PAINS alert and showed similarity with EIDD-1931 and MLN according to the Brenk filter. All selected compounds contain structural alerts for DNA damaging potential and hepatotoxicity risk like MLN and its active form EIDD1931. The details of these structural alerts were shown in Table 4.

All compounds were scanned for the disruption potential on Tox21 pathways [30] and C17 was found to active for p53 tumor suppressor gene pathway like EIDD-1931 (Table 4).

Leading to cardiotoxicity thoroughly QT prolongation related with inhibition of potassium channel encoded by ether-a-go-go-Related gene (hERG) caused withdrawals of many drugs from pharmaceutical market. So, prediction of hERG inhibition is important during drug development to prevent future failure of drug candidates [29]. Also, cardiac complications are one of the main concerns of the COVID 19 viral infections. Consequently, treatment of the disease is expected not to cause cardiotoxicity. Any compounds were not found to exhibit hERG inhibition potential, like MLN and EIDD-1931. None of the compounds were found to have skin sensitisation potential (Table 4). All selected compounds, MLN and EIDD-1931 were found related to hepatotoxicity risk. Drug-induced liver injury is one of the main concerns due to severe hepatic complications of the COVID-19 viral infection and polypharmacy in treatment [31]. Therefore, there is a need for further evaluation in detail if MLN and our designed compounds increase the risks of liver injury.

Inhibition of drug transporters and metabolism enzymes mainly play a role in drug interactions and drug related renal/hepatic toxicity [32,33]. All compounds were found as organic anion transporting polypeptide 2B1 (OATP2B1) and organic anion transporting polypeptide 1B1 (OATP1B1) inhibitors and these features can be increases drug interaction potential of MLN and our designed MLN analogs with the substrates of these OATP transporters. In addition, MLN and EIDD-1931 are cytochrome P450 3A4 (CYP3A4) inhibitors, unlike our designed compounds. 
Table 4. List of predicted toxicity properties (hazard endpoints, Tox21 pathway interaction, target organ toxicity, inhibition properties) of the selected compounds.

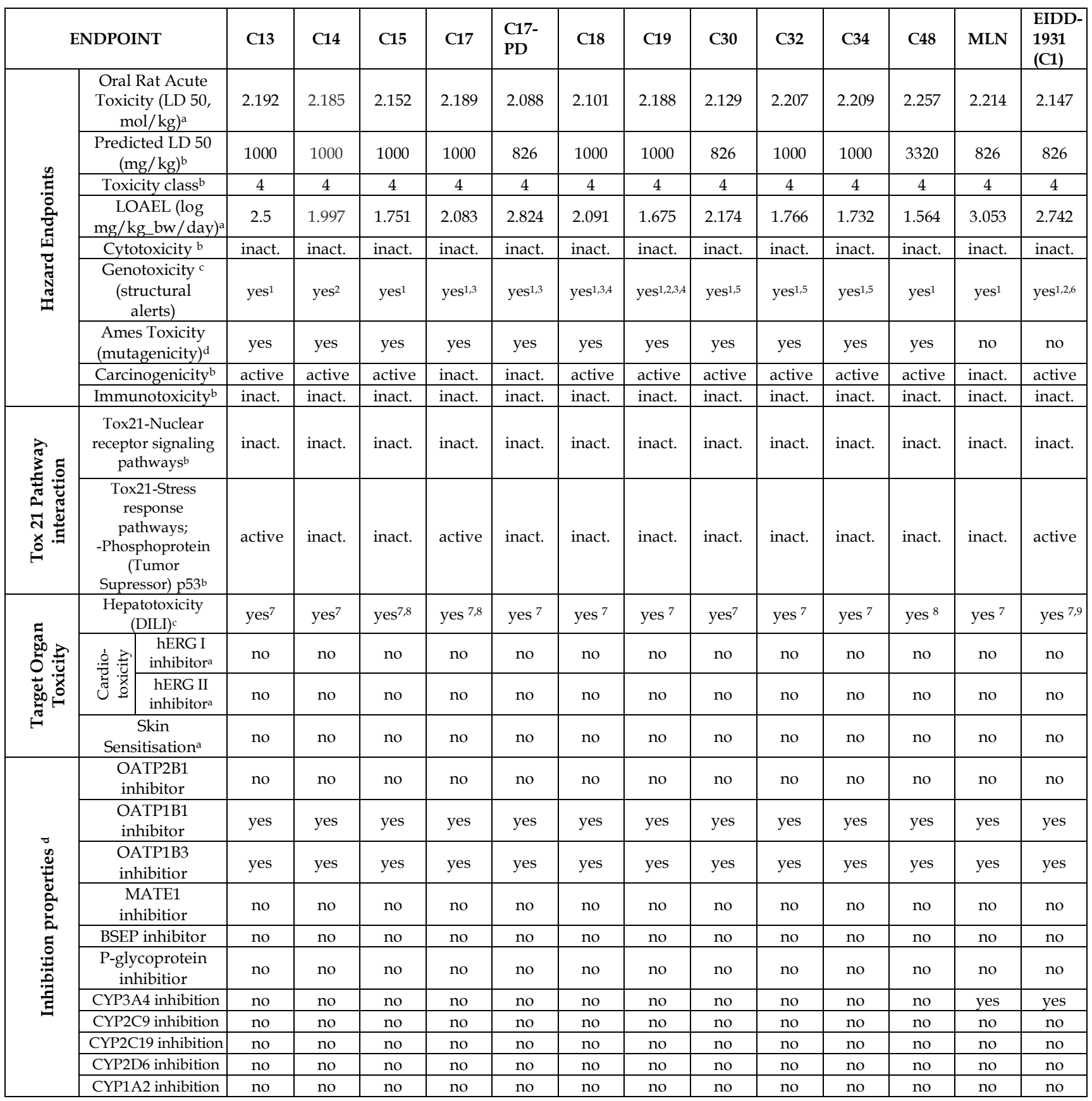

${ }^{a}$ These studies were performed by using online webserver pkCSM; ${ }^{b}$ These studies were performed by using online webserver Protox-II; ${ }^{c}$ These studies were performed by using online webserver eMolTox; ${ }^{d}$ These studies were performed by using online webserver admetSAR. STRUCTURAL ALERTS: ${ }^{1}$ Covalent bind with DNA; ${ }^{2}$ agonist of the 553 signaling pathway; ${ }^{3}$ potential electrophilic agents; ${ }^{4}$ metabolic activation; ${ }^{5}$ PAINS compounds; ${ }^{6}$ Agonist of H2AX. DILI (Drug Induced Liver Injury) ALERTS: ${ }^{7}$ Antagonist of the constitutive androstane receptor (CAR) signaling pathway; ${ }^{8}$ Block OATP1B1 Transporter; ${ }^{9}$ Cytotoxicity in HepG2 cells. Abbreviations: LD50: Letal Dose 50; LOAEL: Low Observed Adverse Effect Level

Painter et al. reported the first phase I clinical trial (clinicalTrials.gov identifier: NCT04392219) results as MLN present good tolerability and dose-proportional pharmacokinetics which is not affected by food consumption following administration to healthy volunteers [34]. According to another Phase I clinical trial (clinicalTrials.gov identifier: NCT04746183) results, MLN was reported as safe and well tolerated and recommended $800 \mathrm{mg}$ twice daily dose for 5 days for Phase II evaluation by Khoo et al. [34]. Any serious adverse effect was not reported by both phase I clinical trials and nowadays phase II/III clinical trials (clinicalTrials.gov identifiers: NCT045755597, NCT045755584, NCT04405570, NCT04939428, etc.) of MLN are ongoing (https:/ / clinicaltrials.gov). Therefore, MLN is a promising oral antiviral agent against SARS-CoV-2, 
fifty four designed analog of MLN were tested in silico for RdRp inhibition, bioavailability, ADME and safety profile and compared with MLN. The ADME/T profile of C17 and C17_PD, which is more potent than EIDD-1931 and MLN, respectively for the target site of the SARS-CoV-2 RdRp enzyme were found to be similar to MLN and its active form EIDD-1931.

\section{CONCLUSION}

In this study, the fifty-four MLN analogues, including compound C13 the previously reported as antiCOVID-19 agent were evaluated against SARS-CoV-2 RdRp using computer-aided methods. Our docking results supported that binding affinity of C13 against related enzyme is higher than MLN like in the literature. Among the designed MLN analogs compound C17 (-7.3 kcal/mol binding energy value) has been found to have the highest affinity even more than compounds C13 and C1. According to the molecular docking studies selected ten compounds with the highest potential enzyme inhibition including C17 are predicted to have a similar bioavailability and safety profile to MLN. Therefore, future studies (in vitro or in vivo) are necessary to confirm the antiviral activity of the $\mathbf{C 1 7}$ by various prodrug forms.

\section{MATERIALS AND METHODS}

\subsection{Molecular docking studies}

\subsubsection{Preparation on enzyme}

The structure of SAR-CoV-2 RNA-dependent RNA polymerase (pdb code: 6m71) [35] was downloaded from the RCSB website with a reported resolution of $2.90 \AA$, and used as a target for the novel molnupiravir analogs. RNA Dependent RNA polymerase is a multimeric protein and the selected crystalized structure is completed by attachment of three additional protein peptides (nsp7-nsp8, and one additional nsp8) to the core polymerase which is chain $\mathrm{A}$ and contains 851 amino acids residues. In this study, we prepared the structures of RdRp without cofactors to investigate the binding affinity of the drugs. This structure without crystallized ligand was protonated using the The AutoDock Tools program and thereafter the obtained structure was energy-minimized.

\subsubsection{Preparation of ligands}

Besides C1 (EIDD-1931), MLN and monophosphate derivatives of related compounds, the designed MLN analogs, compounds C2-C55 were drawn by using the Spartan 04 software[36] (SPARTAN 04, Wavefunction, Inc., Irvine, USA) and optimized for each compound with the semi-empirical PM3 method. For each compound, the most stable conformation was selected for using in docking calculation and these pdb files converted to pdbqt files with The AutoDock Tools program [37].

\subsubsection{Docking studies and analysis}

AutoDock Vina software [38] was used for the designed compounds into the SARS-CoV-2 RdRp structures docking calculations. The results files were analyzed using Accelrys Discovery Studio Visualizer 4.0 program. The size of grid box was detected 30x30x30 points in $x$ (114.52), $y$ (114.11), and $z$ (122.91) dimensions was built; appropriate to the positions of active site residues Asp760 and Asp761 in the 6m71 structure[39]. The Vina parameter "exhaustiveness" was set to the value of 10, besides a grid spacing of 0.375 $\AA$ was employed for the calculation of the energetic map of this enzyme. During the docking studies, we used flexible ligand in rigid protein. As a result of the calculation, 9 different conformations in which the ligand can bind to the enzyme were obtained, and this process was repeated three times for each ligand. For each compound, binding affinities of 27 conformations in total were obtained and selected the ten best active compounds after evaluation of these data.

\subsection{Analysis of bioavalibilty, drug-likeness and ADMET profile}

\subsubsection{Analysis of bioavailability and drug-likeness}

The evaluation of pharmacokinetic properties of a new drug candidate is a major step for the drug discovery and development process. SAR and QSAR models provide in silico rapid and accurate evaluation of the candidates and selection of the molecule that present better characteristic properties in early stages of drug development. The freely accessible servers including Swiss-ADME (http://www.swissadme.ch/), pkCSM (http://biosig.unimelb.edu.au/pkcsm) and Molinspiration (https://www.molinspiration.com/), 
and also software called OSIRIS Datawarrior software were used to predict the molecular and ADME profile of the selected compounds that highest interacted with the active site of the SARS-CoV-2 RdRp enzyme.

\subsubsection{Analysis of ADME and toxicity profiles}

Computational based in silico toxicity measurement has been widely used due to their accuracy, rapidity, accessibility, which can provide information about any compound. To virtually identify the toxicity and adverse effect of the selected compounds that highest interacted with the active site of the SARS-CoV-2 RdRp enzyme, we used freely accessible online web servers including pkCSM, eMolTox (https://xundrug.cn/moltox), admetSAR 2.0 (http://lmmd.ecust.edu.cn/admetsar2/) and ProTox-II (https://tox-new.charite.de/protox_II/). Each tool was used to predict several safety parameters such as acute toxicity, cytotoxicity, carcinogenicity, mutagenicity, immunotoxicity, target organ toxicity, structural alerts related to different toxic effect mechanisms and inhibition potential of the compounds to the several metabolism enzymes and drug transporters.

Author contributions: Concept - I.K., N.K.; Design - İ.K., N.K., T.Y.; Supervision - İ.K.; Data Collection and/or Processing - İ.K., N.K., T.Y.; Analysis and/or Interpretation - N.K., T.Y.; Literature Search - İ.K., N.K., T.Y.; Writing N.K., T.Y., İ.K.; Critical Reviews - N.K., T.Y., İ.K.

Conflict of interest statement: The authors declared no conflict of interest in the manuscript.

Appendix A. Supplementary Material

Supplementary material related to this article can be accessed at https://dx.doi.org/10.29228/jrp.93 .

\section{REFERENCES}

[1] World Health Organization. Coronavirus disease (COVID-19) pandemic n.d. https://www.who.int/emergencies/diseases/novel-coronavirus-2019 (accessed October 2, 2021).

[2] Walensky RP, Walke HT, Fauci AS. SARS-CoV-2 Variants of Concern in the United States-Challenges and Opportunities. JAMA. 2021; 325(11): 1037-1038. [CrossRef]

[3] Parvez MSA, Karim MA, Hasan M, Jaman J, Karim Z, Tahsin T, Hasan MN, Hosen MJ. Prediction of potential inhibitors for RNA-dependent RNA polymerase of SARS-CoV-2 using comprehensive drug repurposing and molecular docking approach. Int J Biol Macromol. 2020; 163: 1787-1797. [CrossRef]

[4] Elfiky AA. Ribavirin, Remdesivir, Sofosbuvir, Galidesivir, and Tenofovir against SARS-CoV-2 RNA dependent RNA polymerase (RdRp): A molecular docking study. Life Sci. 2020; 253: 117592. [CrossRef]

[5] Tian L, Qiang T, Liang C, Ren X, Jia M, Zhang J, Li J, Wan M, YuWen X, Li H, Cao W, Liu H. RNA-dependent RNA polymerase (RdRp) inhibitors: The current landscape and repurposing for the COVID-19 pandemic. Eur J Med Chem. 2021; 213: 113201. [CrossRef]

[6] Wahl A, Gralinski LE, Johnson CE, Yao W, Kovarova M, Dinnon KH 3rd, Liu H, Madden VJ, Krzystek HM, De C, White KK, Gully K, Schäfer A, Zaman T, Leist SR, Grant PO, Bluemling GR, Kolykhalov AA, Natchus MG, Askin FB, Painter G, Browne EP, Jones CD, Pickles RJ, Baric RS, Garcia JV. SARS-CoV-2 infection is effectively treated and prevented by EIDD-2801. Nature. 2021; 591(7850): 451-457. [CrossRef]

[7] Zhou S, Hill CS, Sarkar S, Tse LV, Woodburn BMD, Schinazi RF, Sheahan TP, Baric RS, Heise MT, Swanstrom R. $\beta$ d-N4-hydroxycytidine Inhibits SARS-CoV-2 through lethal mutagenesis but is also mutagenic to mammalian cells. J Infect Dis. 2021; 224(3): 415-419. [CrossRef]

[8] Kabinger F, Stiller C, Schmitzová J, Dienemann C, Kokic G, Hillen HS, Höbartner C, Cramer P. Mechanism of molnupiravir-induced SARS-CoV-2 mutagenesis. Nat Struct Mol Biol. 2021; 28(9): 740-746. [CrossRef]

[9] Rosenke K, Hansen F, Schwarz B, Feldmann F, Haddock E, Rosenke R, Barbian K, Meade-White K, Okumura A, Leventhal S, Hawman DW, Ricotta E, Bosio CM, Martens C, Saturday G, Feldmann H, Jarvis MA. Orally delivered MK-4482 inhibits SARS-CoV-2 replication in the Syrian hamster model. Nat Commun. 2021; 12(1): 2295. [CrossRef]

[10] Menéndez-Arias L. Decoding molnupiravir-induced mutagenesis in SARS-CoV-2. J Biol Chem. 2021;297(1):100867. [CrossRef]

[11] Bonnac LF, Mansky LM, Patterson SE. Structure-activity relationships and design of viral mutagens and application to lethal mutagenesis. J Med Chem. 2013; 56(23): 9403-9414. [CrossRef] 
[12] Hollecker L, Choo H, Chong Y, Chu CK, Lostia S, McBrayer TR, Stuyver LJ, Mason JC, Du J, Rachakonda S, Shi J, Schinazi RF, Watanabe KA. Synthesis of $\beta$-enantiomers of N4-hydroxy-3' -deoxypyrimidine nucleosides and their evaluation against bovine viral diarrhoea virus and hepatitis $C$ virus in cell culture. Antivir Chem Chemother. 2004; 15(1): 43-55. [CrossRef]

[13] Matthes E, Funk A, Krahn I, Gaertner K, von Janta-Lipinski M, Lin L, Will H, Sirma H. Strong and selective inhibitors of hepatitis B virus replication among novel N4-hydroxy- and 5-methyl- $\beta$-L-deoxycytidine analogues. Antimicrob Agents Chemother. 2007; 51(7): 2523-2530. [CrossRef]

[14] Sun L, Peng Y, Yu W, Zhang Y, Liang L, Song C, Hou J, Qiao Y, Wang Q, Chen J, Wu M, Zhang D, Li E, Han Z, Zhao Q, Jin X, Zhang B, Huang Z, Chai J, Wang JH, Chang J. Mechanistic Insight into Antiretroviral Potency of 2'Deoxy-2'- $\beta$-fluoro-4'-Azidocytidine (FNC) with a Long-Lasting Effect on HIV-1 Prevention. J Med Chem. 2020; 63(15): 8554-8566. [CrossRef]

[15] Wang G, Smith DB, Beigelman L, Deval J, Prhavc M. Preparation of substituted nucleosides, nucleotides and analogs thereof as antiviral agents. US 20150051167 A1, 2015.

[16] Lili Y, Shaochun L. Substituted N4-hydroxycytidine derivatives and prodrug thereof for anti-novel coronavirus therapy. CN111548384A, 2020.

[17] Painter GR, Guthrie DB, Bluemling GR, Natchus MR. Preparation of N4-hydroxycytidine and derivatives and antiviral uses related thereto. WO 2017156380 A1, 2017.

[18] Esam Z, Akhavan M, lotfi M, Bekhradnia A. Molecular docking and dynamics studies of Nicotinamide Riboside as a potential multi-target nutraceutical against SARS-CoV-2 entry, replication, and transcription: A new insight. J Mol Struct. 2022; 1247: 131394. [CrossRef]

[19] Raies AB, Bajic VB. In silico toxicology: computational methods for the prediction of chemical toxicity. Wiley Interdiscip Rev Comput Mol Sci. 2016; 6(2): 147-172. [CrossRef]

[20] Lipinski CA, Lombardo F, Dominy BW, Feeney PJ. Experimental and computational approaches to estimate solubility and permeability in drug discovery and development settings. Adv Drug Deliv Rev. 1997; 23 : 3-25. [CrossRef]

[21] Muegge I, Heald SL, Brittelli D. Simple selection criteria for drug-like chemical matter. J Med Chem. 2001; 44(12): 1841-1846. [CrossRef]

[22] Cheng F, Li W, Zhou Y, Shen J, Wu Z, Liu G, Lee PW, Tang Y. AdmetSAR: A comprehensive source and free tool for assessment of chemical ADMET properties. J Chem Inf Model. 2012; 52(11): 3099-3105. [CrossRef]

[23] Pires DEV, Blundell TL, Ascher DB. pkCSM: Predicting small-molecule pharmacokinetic and toxicity properties using graph-based signatures. J Med Chem. 2015; 58(9): 4066-4072. [CrossRef]

[24] Daina A, Michielin O, Zoete V. SwissADME: A free web tool to evaluate pharmacokinetics, drug-likeness and medicinal chemistry friendliness of small molecules. Sci Rep. 2017; 7: 1-13. [CrossRef]

[25] Sander T, Freyss J, Von Korff M, Rufener C. DataWarrior: An open-source program for chemistry aware data visualization and analysis. J Chem Inf Model. 2015; 55(2): 460-473. [CrossRef]

[26] Lin JH, Yamazaki M. Role of P-Glycoprotein in Pharmacokinetics. Clin Pharmacokinet. 2003; 42(1): 59-98. [CrossRef]

[27] Hubatsch I, Ragnarsson EGE, Artursson P. Determination of drug permeability and prediction of drug absorption in Caco-2 monolayers. Nat Protoc. 2007; 2(9): 2111-2119. [CrossRef]

[28] van de Waterbeemd H, Gifford E. ADMET in silico modelling: Towards prediction paradise? Nat Rev Drug Discov. 2003; 2(3): 192-204. [CrossRef]

[29] Miyagawa M. Globally harmonized system of classification and labelling of chemicals (GHS) and its implementation in Japan. Nihon Eiseigaku Zasshi. 2010; 65(1): 5-13. [CrossRef]

[30] Toxicology Testing in the 21st Century (Tox21) n.d. https://www.epa.gov/chemical-research/toxicology-testing21st-century-tox21 (accessed October 6, 2021).

[31] Vitiello A, La Porta R, D'Aiuto V, Ferrara F. The risks of liver injury in COVID-19 patients and pharmacological management to reduce or prevent the damage induced. Egypt Liver J. 2021; 11: 11. [CrossRef]

[32] Ivanyuk A, Livio F, Biollaz J, Buclin T. Renal Drug Transporters and Drug Interactions. Clin Pharmacokinet. 2017; 56(8): 825-892. [CrossRef] 
[33] Ogu CC, Maxa JL. Drug Interactions Due to Cytochrome P450. Baylor Univ Med Cent Proc. 2000; 13: $421-423$. [CrossRef]

[34] Painter WP, Holman W, Bush JA, Almazedi F, Malik H, Eraut NCJE, Morin MJ, Szewczyk LJ, Painter GR. Human Safety, Tolerability, and Pharmacokinetics of Molnupiravir, a Novel Broad-Spectrum Oral Antiviral Agent with Activity Against SARS-CoV-2. Antimicrob Agents Chemother. 2021; 65(5): e02428-20. [CrossRef]

[35] Gao Y, Yan L, Huang Y, Liu F, Zhao Y, Cao L, Wang T, Sun Q, Ming Z, Zhang L, Ge J, Zheng L, Zhang Y, Wang H, Zhu Y, Zhu C, Hu T, Hua T, Zhang B, Yang X, Li J, Yang H, Liu Z, Xu W, Guddat LW, Wang Q, Lou Z, Rao Z. Structure of RNA-dependent RNA polymerase from 2019-nCoV, a major antiviral drug target 2020 (preprint). [CrossRef]

[36] Stewart JJP. Optimization of parameters for semiempirical methods V: Modification of NDDO approximations and application to 70 elements. J Mol Model. 2007; 13(12): 1173-1213. [CrossRef]

[37] Morris GM, Huey R, Lindstrom W, Sanner MF, Belew RK, Goodsell DS, Olson AJ. Software News and Updates Gabedit - A Graphical User Interface for Computational Chemistry Softwares. J Comput Chem. 2009; 30: 174-182. [CrossRef]

[38] Oleg T, J. OA. AutoDock Vina: Improving the speed and accuracy of docking with a new scoring function, efficient optimization, and multithreading. J Comput Chem. 2010; 31(2): 455-461. [CrossRef]

[39] Ahmad J, Ikram S, Ahmad F, Rehman IU, Mushtaq M. SARS-CoV-2 RNA Dependent RNA polymerase (RdRp) - A drug repurposing study. Heliyon. 2020; 6(7): e04502. [CrossRef] 